\title{
The Study of Acupoint Stimulation using Functional Magnetic Resonance Imaging: A Review
}

\author{
Anthony Butler and Tina Huang
}

\begin{abstract}
In recent research on acupuncture there has been much use of magnetic resonance imaging (MRI), particularly the form of MRI that detects brain activity rather than brain structure. This review surveys the literature concerned with one particular aspect of acupuncture research: the authenticity of acupoints. Needling inevitable causes brain activity and researchers have striven to see whether needling an acupoint traditionally linked to one particular organ or physiological process, as distinct from needling elsewhere, causes brain activity associated with that organ or physiological process. The early, positive work, using the zhiyin eye acupoint on the foot and scanning for activity in the occipital lobes of the brain (associated with vision) was partially retracted. Subsequent work has produced mixed results, some positive, some negative. One fairly definite result is that an acupoint is probably an area of the skin rather than a single point. The ambiguity of the data is partly explained by the enormous experimental difficulties experienced in using MRI for this type of work and the challenge of finding a way of administering authentic sham acupuncture. The need for further research is emphasised.
\end{abstract}

\section{Keywords}

acupuncture, acupoints, sham acupuncture, brain activity

That acupuncture is a valuable therapy in the treatment of certain conditions is not in doubt. As evidence of its authenticity, a meta-analysis by Ernst and co-workers ${ }^{1}$ using 22 random-controlled trials from researchers all over the world, comes down firmly in favour of acupuncture's value in the management of lower back pain. There is similar evidence to support the use of acupuncture in the relief of neck pain. ${ }^{2}$ Whether acupuncture can treat all the conditions proposed by its more enthusiastic proponents is less certain. ${ }^{3}$ That said, its success in treating even a limited number of conditions means that it must be taken seriously. However, an explanation of why it works, in terms of Western neuroscience, is something of a challenge. Some would argue that it

\footnotetext{
1 Manheimer et al. 2006.

2 Trinh et al. 2007.

3 Ernst 2006.
} 
can be understood only in terms used by traditional Chinese medicine: the balance of yin and yang, the flow of qi and the wuxing. The 'doctrines' of traditional Chinese medicine are seen as another way of describing the human body, as valid as the current 'doctrines' of Western physiology. Others see the success of acupuncture as a placebo effect. ${ }^{4}$ The use of the term 'placebo' should not be seen as a suggestion that acupuncture is in some way fraudulent, although that was the way the term was first used in medicine. However, the work of Beecher ${ }^{5}$ and others in the 1950s established that a placebo could really heal people. It is now seen as an acceptance that our minds and bodies are so intimately connected that, although we readily recognise that the condition of our bodies influences our mental states, the converse is also true. In the final analysis the most important factor is that the patient feels better.

\section{The advent of imaging}

Modern physiology has been very successful in providing a coherent if, as yet, incomplete, account of why the human body works the way it does, leading to the outstanding success of modern, Western medicine even in cultures where traditional Chinese medicine is practised. ${ }^{6}$ It is not unreasonable, therefore, to see whether acupuncture fits into the pattern that has been uncovered. Much effort was expended, in both China and the West, particularly during the period 1950-1990, trying to find a neurochemical basis for acupuncture ${ }^{7}$ by studying the opioids released on needling acupoints. Some studies used animal models and the extension of such studies to humans is fraught with difficulty. Han and Terenius' careful perusal of the substantial number of studies done until that time did not provide a clear picture of what, in terms of neurochemicals produced, needling of acupoints does that is distinctive. Much of the work was well conceived but analytical procedures at that time were insufficiently sensitive to lead to unambiguous conclusions.

Experimental work on acupuncture was dramatically changed when a number of brain imaging techniques became generally available. Here were nonintrusive procedures that could assess the effect upon brain activity of needling an acupoint. The subject of imaging was reviewed in 2005 by Lewith, White and Pariente ${ }^{8}$ but much work has been done since then. It is a complex topic

\footnotetext{
4 Purves et al. 1997.

Beecher 1955.

Le Fanu 1999.

Han and Terenius 1982.

8 Lewith et al. 2005.
} 
and the current review is concerned with only one imaging technique, Magnetic Resonance Imaging (MRI). This technique was considered, along with others, in a review of 2007 by Dhond et al. ${ }^{9}$ This review is particularly authoritative and should be consulted by all serious students of acupuncture but requires considerable background knowledge of brain structure. The present review reports the most recent work on MRI and the needling of acupoints and is aimed more at the general reader.

It is not out of place to say a little about the physical basis of the technique of MRI. How placing an object in a strong magnetic field can lead to the generation of a $3 \mathrm{D}$ image of that object is almost as mystifying as acupuncture itself. A full explanation ${ }^{10}$ requires an understanding of quantum physics, which not all readers may have, but a short account may be beneficial in alerting the reader to the enormous potential of MRI but also registering the considerable difficulties experienced in its use.

The precursor of MRI is Nuclear Magnetic Resonance (NMR) spectroscopy, which completely revolutionised the study of chemistry in the 1960s. For NMR spectroscopy the chemical sample is placed in a very strong, but completely uniform, magnetic field (essentially between the poles of a magnet). Any chemical sample containing hydrogen, when placed in such a magnetic field, will absorb radio-frequency radiation of one, and only one, frequency, hence the use of the term resonance. The exact frequency depends upon the strength of the magnetic field. Also, the amount of radiation absorbed depends upon the numbers of hydrogen atoms in the sample. How that leads to a form of chemical spectroscopy need not concern us but, for imaging purposes, the innovation was to put the sample, not in a uniform magnetic field but, in a field with a magnetic strength gradient on it. Then hydrogen atoms in different parts of the sample will resonate at different frequencies and the amount of radiation absorbed at each frequency reflects the number of hydrogen atoms at that location. The most common form of hydrogen in the human body is as water $\left(\mathrm{H}_{2} \mathrm{O}\right)$. Thus, if a human limb is placed in a gradated magnetic field and irradiated with radio frequencies in the appropriate range, water in different parts of the limb will absorb radiation of different frequencies and the amount of radiation of each frequency absorbed depends on the amount of water at that location. Thus a water density map in one dimension is obtained. By rotating the magnet a similar density map is obtained along another dimension. By repeated use of this procedure and with the use of an enormous amount of computing, a 3D picture of water concentration in the

9 Dhond et al. 2007.

10 Brown and Semelka 2010. 
limb results. The whole process is nonintrusive. Different types of soft tissue vary in their water content and so MRI can distinguish between them. This is enormously important in the detection of tumours. The technique was initially known as NMR imaging but it was felt that the term 'nuclear' would frighten the patient and it was dropped.

Modern MRI is considerably more complex than what has been described and depends greatly upon the power of modern computers and the development of new, magnetic materials. The challenge is to improve contrast between different types of soft tissue and a major factor there is the strength of the magnetic field. The unit of magnetic field strength is the tesla (T) and early MRI instruments used fields of $0.1 \mathrm{~T}$. With fields of $0.5 \mathrm{~T}$ the amount of detail in an image, particularly if all other parameters are optimised, is truly remarkable. With modern research instruments that have fields as strong as $7 \mathrm{~T}$ the resolution is so good that structural features of, say, a living brain that have not previously been described can be discerned. MRI has undoubtedly revolutionised both diagnostic medicine and neuroscience research.

So far what has been described gives a static, anatomical picture of organs of the human body but it is possible to do more than this. Most tissue water is static but blood, which is flowing rather than static, shows up differently on an MR image, giving rise to functional MRI (fMRI). This refinement can be extended in a way that is particularly useful in detecting brain activity. Stimulated regions of the brain experience an increase in blood supply with increased delivery of oxygenated haemoglobin and decreased supply of deoxygenated haemoglobin. The two forms of haemoglobin have different magnetic properties and this can be used to enhance an MR image. The effect is known as the blood-oxygenation-level-dependent (BOLD) effect. ${ }^{11}$ In a typical experiment a set of images is obtained for a patient whose brain is being stimulated in some way (for example, by shining a light into the eye of the patient and focussing on those parts of the brain concerned with vision) and another set without the stimulation. The latter set is subtracted from the former and what remains is the fMRI BOLD effect. This is then superimposed on an anatomical image of the brain to give a picture of the brain with certain regions highlighted. The result is a map, not of brain structure, but of brain activity. It is BOLD fMRI that has been mainly used in the study of acupuncture, requiring this rather long introduction to the subject.

There are dangers and difficulties in the use of MRI. The equipment is very expensive and requires a skilled operator. The patient is placed in a tunnel to get the brain between the poles of a magnet and many experience severe

${ }^{11}$ DeYoe et al. 1994. 
claustrophobia and further testing is impossible. Many scans are necessary to get a good image and the total acquisition time is generally about 20 minutes and during that time the patient must remain completely still otherwise the image becomes blurred. For some people this is impossible. Metallic implants distort the magnetic field, making imaging very difficult. Even if all these matters are satisfactorily dealt with, the technique of MR imaging remains a difficult one.

\section{Needling and imaging}

The seminal paper on assessing the effect by MRI of needling an acupoint on brain activity appeared in 1998 in no less a journal than the Proceedings of the National Academy of Sciences USA. ${ }^{12}$ The vision-related acupoint zhiyin (至陰) is located, according to classical acupuncture practice, in the lateral aspect of the foot and, when needled, there is stimulation of the occipital lobes of the brain, as observed by BOLD fMRI. The same stimulation is observed when the eye is stimulated by light. Results from 12 volunteers yielded very clean data. As a control, the needling of nonacupoints, 2 to $5 \mathrm{~cm}$ away from zhiyin (sham acupuncture,) was found not to stimulate the occipital lobes. This work appeared to constitute very strong evidence for linking a specific point on the body, via neural activity, to a particular organ. However, in 2006 this paper was partially retracted by some of the authors, but not by others. The reason for the retraction is given as 'Accumulating evidence suggests that the central nervous system is essential for processing these effects, via its modulation of the autonomic nervous system, neuro-immune system and hormone regulation. ${ }^{13}$ Further comments concern acupoint specificity with regard to pain and analgesia and their revised conclusion is that there is no such specificity. In the retraction they do not mention zhiyin and the occipital lobes but clearly the paper can no longer be regarded as providing authoritative evidence for the specificity of acupoints.

Before retraction, a related study ${ }^{14}$ of laser acupuncture at zhiyin on the left foot gave support for the original view. For sham laser acupuncture the laser was placed on the skin at zhiyin but not switched on. In this study there were 10 volunteers in the verum and sham groups. When the brain images were compared it was found that the results were similar to those of Cho et al. ${ }^{15}$

12 Cho et al. 1998.

13 Cho et al. 2006, p. 10527.

${ }_{14}$ Golaszewski et al. 2001.

15 Cho et al. 1998. 
with stimulation of the occipital lobes in the left hemisphere, but not in the right. Similar results were obtained using electro-acupuncture as well as conventional acupuncture with four vision-related acupoints. ${ }^{16}$ However, in a very thorough study Kong et al. ${ }^{17}$ used electro-acupuncture at two vision-related acupoints on the leg, kunlun (崑崙) and guangming (光明) and at nearby sham acupoints. Electro-acupuncture at both the verum and sham acupoints had a similar effect on the occipital cortex, providing no support for the specificity of acupoints. There is no doubt that these data are at odds with previous work. Li $e t a l{ }^{18}$ comment on the contradictory nature of research findings, explaining it in terms of the complexity of the situation and the experimental difficulties encountered when attempting imaging, a view given added significance by the later retraction of the original work. Further discussion is postponed until more results have been described.

Other workers have compared the neural effects of needling at sham and verum acupoints not related to vision. The wrist acupoint neiguan (內關 is needled to prevent nausea and vomiting. Yoo et al. ${ }^{19}$ found that needling neiguan stimulated a number of areas of the brain but some of this stimulation was reckoned to be due to the volunteer listening to the noise of the scanner in the MRI machine. Sham acupuncture, either sensory stimulation of the skin without penetration or penetration away from neiguan, also produced extensive neural stimulation but there were consistent differences between verum and sham acupuncture. Important cerebellar areas stimulated only by verum acupuncture at neiguan include the left superior frontal gyrus, anterior angulate gyrus and parts of the thalamus. Clearly the situation is more complex than with the vision-related acupoint selected by Cho et al. ${ }^{20}$ but that could be because the physiological processes associated with vomiting and nausea are more extensive than those involved in vision. In a similar way, images resulting from the needling of taichong (太衝) on the foot and hegu (合谷) on the hand of 37 healthy volunteers revealed a pattern of brain activity not found on sham needling. ${ }^{21}$

Interesting data ${ }^{22}$ were obtained when brain images seen on needling at two acupoints on the same meridian were compared with needling an acupoint on an entirely different meridian of no therapeutic relevance. The acupoints

\footnotetext{
16 Li et al. 2003.

17 Kong, Kaptchuk, Webb et al. 2009.

18 Li et al. 2008.

19 Yan et al. 2005.

${ }^{20}$ Cho et al. 1998

21 Yan et al. 2005.

22 Bai et al. 2010.
} 
neiguan and daling (大陵) are both on the pericardium channel on the arm whereas guangming is located on the gallbladder channel on the leg. Only small differences were seen in the neural effects of needling neiguan and daling and stimulation was limited to the cerebrocerebellum and subcortical ares, while needling at guangming produced a more widely spatial distribution of neural responses. These results are consistent with the existence of meridians but hardly confirm their reality. A related study of neiguan needling by Napadow et al. ${ }^{23}$ showed that verum acupuncture produced more varied and persistent brain responses than sham (nonpenetration) acupuncture at the same acupoint. A similar result was reported by Wang et al. ${ }^{24}$ for the needling of chongyang (衝陽) and zusanli (足三里) acupoints, with stimulation of random sham acupoints as a control. The size, signal intensity and number of brain areas showing activation were fewer in members of the control group than with those undergoing verum acupuncture.

During acupuncture the needle is often manipulated. Images were obtained of rotation of the needle at acupoints, taichong (太衝) and quixu (丘噓), both in the metatarsal region of the foot, and at a sham acupoint on the dorsum of the foot. The conclusion of this study ${ }^{25}$ was that rotation caused an increase in activation of certain parts of the brain but no such effects were observed on needle rotation at the sham acupoint, supporting the idea that there is something special about traditional acupoints. In a comparison of relatively superficial and deep needling of the right foot acupoint hegu, little difference was noted in the brain images between the two, consistent with the clinical success reported by both Chinese (deep needling) and Japanese (superficial needling) acupuncturists. ${ }^{26}$

\section{Imaging and deqi}

In the practice of acupuncture the needling sensation deqi (得气) is considered important by practitioners. The deqi sensation is quite different from the acute pain that is sometimes experienced on needling. Asghar et al. ${ }^{27}$ have used BOLD fMRI to compare the effect of deqi and acute pain on brain activity, using the needling of the right hegu acupoint. A number of brain areas showed activity in both cases but involving significantly different areas.

\footnotetext{
23 Napadow et al. 2009.

24 Wang et al. 2001.

25 Fang et al. 2004.

26 MacPherson et al. 2008.

27 Asghar et al. 2010.
} 
Confirmation of these observations comes from a further $\operatorname{paper}^{28}$ on acupuncture and pain reduction described the neural effects of needling hegu, zusanli and taichong and compared them with those observed on sham acupuncture (tactile stimulation). Verum acupuncture causes more extensive stimulation of several regions of the brain. There were also differences between subjects experiencing deq $i$ with pain compared with those experiencing deqi with no pain. Part of this paper is a video, available online to subscribers. The experimental precautions necessary to prevent the subject showing spurious stimulations due to noxious influences (like movement of the experimenter) are noted. Clearly all imaging studies should include a record of the participants' perception of deqi. To complicate the situation further, deqi effects have been found ${ }^{29}$ on needling at sham acupoints. This suggests that needling at what appear to be sham acupoints does not always constitute a good control. Another difficult issue is that the response to acupuncture may vary from person to person and so significant neural effects may diminish if averaged over a group where some participants show a much smaller effect. Thus, to gauge what acupuncture can do, as well as studying groups of subjects, something extra might be learned from studies of one person with whom the effect is pronounced. ${ }^{30}$ Indeed, MacPherson ${ }^{31}$ has argued for an individual-based approach to acupuncture research. With the repeated use of one person expectation becomes a major problem.

\section{Time-dependent imaging}

Yet another complication is that an acupuncture effect may occur or continue after the needle has been removed and this is a common clinical observation. A long-lasting neural effect was observed ${ }^{32}$ after needle manipulation at daling (大陵) had been discontinued. Bai et al..$^{33}$ found, from MRI images, that needling at zusanli produced a neural effect that varied between the early and late parts of the process. Thus, averaging a set of images over time may not give a true picture of the acupuncture process. Rather different time-related neural effects were observed for the electro-acupuncture stimulation at guangming and jiaoxin (交信) on the left leg. The two points displayed very different

\footnotetext{
28 Hui et al. 2010.

29 Hsieh et al. 2001; Vincent and Lewith 1995.

30 Sun et al. 2010.

31 MacPherson 2005.

32 Bai et al. 2010.

33 Bai et al. 2009.
} 
temporal patterns and the authors ${ }^{34}$ suggest the time-related pattern may fix the functional specificity of the acupoint. In a study of needling at neiguan it was found ${ }^{35}$ that verum acupuncture leads to more persistent neural stimulation than sham (non-intrusive cutaneous stimulation). Time variability of neural effects was observed for needling at zusanli in a study by Zhang et al. ${ }^{36}$

Sometimes acupuncture is claimed to cause a rather obvious physical symptom. Needling at erjian (二間) is claimed to stimulate saliva production and is, therefore, a treatment for excessive dryness of the mouth (xerostomia). When examined by $\mathrm{fMRI}$, neural effects accompanied by salivation were observed, but these effects were not observed on needling nearby sham acupoints. ${ }^{37}$

As if acupuncture were not complicated enough to investigate, some brave researchers, in a complex and extensive study, ${ }^{38}$ have shown that expectancy of what effect acupuncture would have can affect both analgesic and neural changes. Positive expectation of acupuncture in response to induced and calibrated pain can not only amplify analgesia, as detected by subjective pain sensory rating changes, but also enhance fMRI signal changes. As do others, this study illustrates well how complex acupuncture research can be.

\section{Summing up}

It is now appropriate to sum up. The aim of BOLD fMRI studies is not to establish the clinical validity of acupuncture but to confirm (or otherwise) the claim, made in traditional acupuncture practice, that there is a connection between, often, remote parts of the body (the acupoints) and the functioning of organs of the body. By the use of fMRI the connection is being made through the brain although this is not, in any way, part of the traditional, theoretical basis of TCM. Inserting a needle into human tissue is almost certain to produce a neural response; the question is whether needling an acupoint elicits a neural response of a special kind. It is clear from the evidence reviewed that we are still a long way from establishing this claim beyond doubt. The clean, unambiguous results reported by Cho et al. ${ }^{39}$ linking needling at zhiyin on the foot with neural activity associated with vision, have been partially retracted and, equally significantly, have not been replicated

34 Liu et al. 2009.

35 Hui et al. 2010.

36 Zhang et al. 2009.

37 Deng et al. 2008.

38 Kong, Kaptchuk, Polich et al. 2009.

${ }^{39}$ Cho et al. 1998. 
firmly by subsequent workers. On the other hand, no damning evidence against the connectivity of acupoints and specific brain areas has been produced. The inherent difficulties associated with the use of fMRI, mentioned earlier, make sure and certain conclusions difficult. The complicating factor of expectancy could induce a feeling of despair in researchers, suggesting that we can never have unambiguous evidence of the status of acupoints, but this is to ignore what positive results have been obtained. One factor that does emerge from the data presented is that sham acupuncture (needling a short distance away from the traditional acupoint) is not quite the cast-iron control that we thought it was. Several studies suggest that the traditional acupoint merely optimises an effect that can be elicited by needling over quite an area. In fact, this is not a new conclusion. ${ }^{40}$ How then do we establish a protocol for genuine sham acupuncture? Until this difficulty has been solved, progress towards certainty will be compromised. Equally, the time dependence of the acupuncture effect means that the time dimension must be taken into account. The use of very high fields (3T and above) shortens acquisition times for MRI imaging and should allow the temporal variation to be explored.

From a clinical prospective analgesia is the condition for which acupuncture is most valuable. Unfortunately, pain produces very complex neural responses ${ }^{41}$ and this makes the needling of pain-relieving acupoints difficult to study by imaging techniques. ${ }^{42}$ The seminal work of $\mathrm{Hiu}^{43}$ indicates that needling of hegu, one of the most frequently used analgesic points, produces widespread effects in the brain and that negative BOLD signals are observed. Although pain relief by acupuncture demands further study, more readily interpreted data might be obtained from the study of relatively simple situations, like the link between zhiyin and stimulation of the occipital lobes.

Although a lack of certainty emerging from 13 years of fMRI studies is disappointing, there is enough positive evidence to give impetus for the continuation of these experimentally demanding studies. A sufficiently large amount of experimental information has been accumulated to provide the direction for further research. Linking data from BOLD fMRI with that from other imaging techniques should move the subject forward. However, many more data are required before we can be certain of the detailed link between needling of traditional acupoints and regionally-specific neural activity. The design of experiments and how data are analysed can be as complex as the physics behind fMRI.

\footnotetext{
40 Stux, Berman and Pomeranz 2003, pp. 42-3.

41 Bornhövd et al. 2002.

42 Wang et al. 2001.

${ }^{43}$ Hui et al. 1997.
} 


\section{References}

Asghar, A.U.R., G. Green, M.F. Lythgoe, G. Lewith and H. MacPherson 2010, 'Acupuncture needling sensation: The neural correlates of deqi using fMRI', Brain Research, 1315: 111118.

Bai, L., W. Qin, J. Tian, P. Liu, L.L. Li, P. Chen, J. Dai, J.G. Craggs, K.M. von Deneen and Y. Liu 2009, 'Time-varied characteristics of acupuncture effects in fMRI studies', Human Brain Mapping, 30: 3445-3460.

Bai, L., H. Yan, L. Li, W. Qin, P. Chen, P. Liu, Q. Gong, Y. Liu and J. Tian 2010, 'Neural specificity of acupuncture stimulation at Pericardium 6: Evidence from an FMRI study', J Magnetic Res Imaging, 31: 71-77.

Beecher, H.K. 1955, 'The powerful placebo', Journal of the American Medical Association' 159, 1602-1606.

Bornhövd, K., M. Quante, V. Glauche, B. Bromm, C. Weiller, and C. Büchel 2002, 'Painful stimuli evoke different stimulus-response functions in the amygdale, prefrontal and somatosensory cortex: a single-trial fMRI study' Brain, 125: 1326-1336.

Brown, M.A. and R.C. Semelka 2010, MRI Basic Principles and Applications, 4th ed., WileyBlackwell, New Jersey.

Cho, Z.H., S.C. Chung, H.J. Lee, E.K. Wong and B.I. Min 2006, 'Retraction.' Proc. Natl. Acad. Sci. USA, 103: 10527.

Cho, Z.H., S.C. Chung, J.P. Jones, J.B. Park, H.J. Park, H.J. Lee, E.K. Wong and B.I. Min 1998, 'New findings of the correlation between acupoints and corresponding brain cortices using functional MRI', Proc. Natl Acad. Sci, USA, 95: 2670-2673.

Deng, G., B.L. Hou, A.I. Holodny and B.R. Cassileth 2008, 'Functional magnetic resonance imaging (fMRI) changes and saliva production associated with acupuncture at LI-2 acupuncture point: a randomised controlled study', BMC Complementary and Alternative Medicine, $8,37-41$.

DeYoe, E.A., P. Bandettini, J. Neitz, D. Muler and P. Winans 1994, 'Functional magnetic resonance imaging of the human brain', Journal of Neuroscience Methods, 54: 171-187.

Dhond, R.P., N. Kettner and V. Napadow 2007, Neuroimaging acupuncture effects in the human brain. J. Alter. Complement. Med., 13: 603-616.

Ernst, E. 2006, 'Acupuncture-a critical analysis', Journal of Internal Medicine, 259: 125-137.

Fang, J.L., T. Krings, J. Weidemann, I.G. Meister and A. Thron 2004, 'Functional MRI in healthy subjects during acupuncture: different effects of needle rotation in real and false acupoints', Neurobiology, 46, 359-362.

Golaszewski, S., C. Siedentopf, C. Kremster, F. Mothaghy, A. Schlager and S. Felber 2001, 'Effects of laser acupuncture on activation within the visual cortex: A functional MRI study', Neuroimage, 13: S978.

Han, J.S. and L. Terenius 1982, 'Neurochemical basis of acupuncture analgesia', Annual Review of Pharmacology and Toxicology, 22: 193-220.

Hsieh, J., C. Tu, F. Chen, M. Chen T. Yeh, H. Cheng, Y. Wu, R. Liu, R. Liu, L. Ho 2001, 'Activation of the hypothalamus characterizes the acupuncture stimulation of the analgesic point in humans: a positron emission tomography study', Neuroscience Letters, 307, 105-108.

Hui, K.K.S., V. Napadow, J. Liu, M. Li, O. Marina, E.K. Nixon, J.D. Claunch, L. LaCount, T. Sporko and K.K. Kwong 2010, 'Monitoring acupuncture effects on human brain by fMRI', Journal of Visualized Experiments, http://www.jove.com/index/Details.slp?ID=1190.

Hui, K.K.S., J. Liu, K.K. Kwong 1997, 'Functional mapping of the human brain during acupuncture with magnetic resoanance imaging somatosensory cortex activation', World J AcupMox, 7, 44-49. 
Kong, J., T.J. Kaptchuk, J.M. Webb, J-T. Kong, Y. Sasaki, G.R.

Kong, J., T.J. Kaptchuk, G. Polich, I. Kirsch, M. Vangel, C. Zyloney, B. Rosen and R.L. Gollub 2009, 'An fMRI study on the interaction and dissociation between expectation of pain relief and acupuncture treatment', Neuroimage, 47: 1066-1076.

Le Fanu. J. 1999, The Rise and Fall of Modern Medicine, Little, Brown \& Co., London.

Lewith, G.T., P.J. White and J. Pariente 2005, 'Investigating acupuncture using brain imaging techniques: The current state of play', Evidence-based Complementary and Alternative Medicine, 2: 315-319.

Li, G., R.T.F. Cheung, Q-Y Ma and E.S. Yang 2003, 'Visual cortical activations on fMRI upon stimulation of vision-implicated acupoints', Brain Imaging, 14: 669-673.

Li, X-Z., X-G. Liu and F-R. Liang 2008, 'Functional brain imaging studies on specificity of meridian and acupoints' Neural Regeneration Research, 3: 777-781.

Liu, P., W. Wei, Y. Zhang, J. Tian, L. Bai., G. Zhou, J. Liu, P. Chen, J. Dai, K.M. von Deneen and Y. Liu 2009, 'Combining spatial and temporal information to explore function-guide action of acupuncture using fMRI', Journal of Magnetic Resonance Imaging, 30: 41-46.

MacPherson, H. 2005, 'Evidence-based acupuncture? A challenge ahead'. Asian Medicine, 1: 149-161.

MacPherson, H., G. Green, A. Nevado, M.F. Lythgoe, G. Lewith, R. Devlin, R. Haselfoot and A.U.R. Asghar 2008, 'Brain imaging of acupuncture: Comparing superficial with deep needling', Neuroscience Letters, 434: 144-149.

Manheimer, E., A. White, B. Berman, K. Forys and E. Ernst 2005 'Meta-analysis: Acupuncture for low back pain, Ann. Intern. Med., 142, 651-663.

Napadow, V., R.P. Dhond, J. Kim, L. LaCount, M. Vangel, R.E. Richards, N. Kettner and K. Park 2009, 'Brain encoding of acupuncture sensation-Coupling on-line with fMRI', Neuroimage, 47: 1055-1065.

Polich, M.G. Vangel, K. Kwong, B. Rosen and R.L. Gollub 2009, 'Functional neuroanatomical investigation of vision-related acupuncture point specificity-A multisession fMRI study', Human Brain Mapping, 30: 38-46.

Purves, D., G.J. Augustine, D. Fitzpatrick, L.C. Katz, A-S. LaMantia and J.O. McNamara 1997, Neuroscience, Sunderland, Sinauer Associates Inc.

Stux, G., B. Berman and B. Pomeranz 2003, Basics of Acupuncture, 5th ed, Springer Medizin Verlag, Heidelberg.

Sun J., W. Qin, M. Dong, K. Yuan, J. Liu, P. Liu, Y. Zhang, K.M. von Deneen and J. Tian 2010, 'Evaluation of group homogeneity during acupuncture stimulation in fMRI studies', Journal of Magnetic Resonance Imaging, 32: 298-305.

Trinh, K., N. Graham, A. Gross, C. Goldsmith, E. Wang, I. Cameron and T. Kay 2007, 'Acupuncture for neck disorders', Spine, 32, 236-243.

Vincent P.A. and G. Lewith 1995, 'Placebo controls for acupuncture studies', Journal of the Royal Society of Medicine, 88: 199-202.

Wang, G.B., C. Liu, L.B. Wu, B. Yan, S.Z. Gao, G.R. Shao, Q.C. Lü (王光涁, 柳骋, 武乐 斌, 阊镔, 高树中, 邵光瑞, 吕庆超) 2001, 'Functional magnetic resonance imaging on acupuncturing yuan-source and he-sea acupoints of stomach meridian of foot-yangmin,' 中国㗨 學科學阮學報 (Zhongguo Yi Xue Ke Xue Yuan Xue Bao), 31, 171-176.

Yan, B., K. Li, J. Xu, W. Wang, K. Li, H. Liu, B. Shan and X. Tang 2005, 'Acupoint-pecific fMRI patterns in human brain', Neuroscience Letters, 383, 236-240.

Yoo, S-S., E-K. Teh, R.A. Blinder and F.A. Jolesz 2004, 'Modulation of cerebellar activities by acupuncture stimulation: evidence from fMRI studies', Neuroimage, 22: 932-940.

Zhang, Y., W. Qin, P. Liu, J. Tian, J. Liang, K.M. von Deneen and Y. Liu 2009, 'An fMRI study of acupuncture using independent component analysis', Neuroscience Letters, 449: 6-9. 